\title{
Low defect densities in molecular beam epitaxial GaAs achieved by isoelectronic In doping
}

Pallab K. Bhattacharya, Sunanda Dhar, ${ }^{\text {a) }}$ Paul Berger, and Feng-Yuh Juang

Solid State Electronics Laboratory, Department of Electrical Engineering and Computer Science, The University of Michigan, Ann Arbor, Michigan 48109

(Received 2 April 1986; accepted for publication 1 July 1986)

We have studied the effects of adding small amounts of In (0.2-1.2\%) to GaAs grown by molecular beam epitaxy. The density of four electron traps decreases in concentration by an order of magnitude, and the peak intensities of prominent emissions in the excitonic spectra are reduced with increase in In content. Based on the higher surface migration rate of In, compared to $\mathrm{Ga}$, at the growth temperatures it is apparent that the traps and the excitonic transitions are related to point defects. This agrees with earlier observations by F. Briones and D. M. Collins [J. Electron. Mater. 11, 847 (1982)] and B. J. Skromme, S. S. Bose, B. Lee, T. S. Low, T. R. Lepkowski, R-Y. DeJule, G. E. Stillman, and J. C. M. Hwang [J. Appl. Phys. 58, $4702(1985)]$.

In, with a slightly larger atomic radius than $\mathrm{Ga}$, has been successfully used to drastically reduce dislocation densities in liquid encapsulation Czochralski (LEC) GaAs, ${ }^{1-3}$ and this behavior has been explained on the basis of a solid solution hardening model. ${ }^{4}$ The reduction in dislocation density has been correlated with improvement in material properties and the characteristics of field-effect transistors made by direct implantation into these materials. ${ }^{5,6} \mathrm{Im}$ provement in the electrical and optical properties of isoelectronically doped liquid phase epitaxial GaAs:In and InP:Ga(As) and metalorganic vapor phase epitaxial GaAs:In has been recently demonstrated by Beneking et $a l^{7-10}$

The effects of isoelectronic doping during molecular beam epitaxial (MBE) growth of III-V semiconductors have not been reported. Recent calculations by Singh" show that at a growth temperature of $600^{\circ} \mathrm{C}$ the In adatom which arrives from the vapor and forms two In-As bonds with the growing surface has a surface migration rate of $2 \times 10^{5} \mathrm{hops} / \mathrm{s}$. The corresponding Ga migration rate under identical conditions is $10^{4}$ hops/s. It is, therefore, most likely that the fast migrating In surface atoms, with a weaker InAs bond than that for Ga-As, will occupy normally vacant Ga sites in the growing crystals and thereby lower the density of such vacancy-related defects. In the present study we have determined the variation of the density of deep level traps and of defect-related centers in the photoluminescence excitonic spectra with the amount of In in the solid solution.

A series of GaAs layers without and with small amounts of In doping was grown by molecular beam epitaxy on undoped semi-insulating and Si-doped $n^{+}$substrates $\left(n \sim 5 \times 10^{18} \mathrm{~cm}^{-3}\right)$ under As-stabilized conditions $\left(\mathrm{As}_{4} / \mathrm{Ga}=15\right)$ with a substrate temperature of $560^{\circ} \mathrm{C}$. Typical layer thicknesses varied from 2 to $5 \mu \mathrm{m}$. The In content $(x)$ was determined from the fluxes and was confirmed by low-temperature photoluminescence measurements using the relation: $x=-\Delta h v / 1.505$ fitting. In the crystals measured in the course of this study, the In content varied from 0 to $1.2 \%$. All the undoped crystals were $p$ type with $\left(N_{a}-N_{d}\right)=(1-5) \times 10^{15} \mathrm{~cm}^{-3}$. For the study of

\footnotetext{
- Permanent address: Institute of Radiophysics and Electronics, The University of Calcutta, 92, Acharya P.C. Ray Road, Calcutta 700 009, India.
}

deep level traps, a $0.5-\mu \mathrm{m}$ Be-doped $p^{+}\left(2 \times 10^{18} \mathrm{~cm}^{-3}\right)$ top layer was grown to facilitate the formation of ohmic contacts. Junction diodes with $250 \mu \mathrm{m}$ diameter were formed by photolithography and lift-off techniques. Low-temperature photoluminescence measurements were made with the samples mounted in a strain-free manner on a cold finger. Excitation was provided by an $\mathrm{Ar}^{+}$laser $(5145 \AA)$. The luminescence from the sample was analyzed by a 1-m Jarell-Ash scanning spectrometer and detected by a cooled $S 1$ photomultiplier. The spectra were recorded after lock-in amplification. The luminescence measurements were made on samples without the top $p^{+}$layer.

It is known that MBE GaAs contains several electron traps of which the traps labeled $M 1, M 3$, and $M 4^{12,13}$ occur more consistently. On the other hand, the occurrence of hole traps is not so consistent, and their presence in MBE GaAs is mostly related to dopants or $\mathrm{Fe}$ and $\mathrm{Cu}$ impurities. ${ }^{13,14}$ In a series of lightly Si-doped $\left[\sim(1-5) \times 10^{15} \mathrm{~cm}^{-3}\right]$ samples, optical deep level transient spectroscopy (ODLTS) mea-

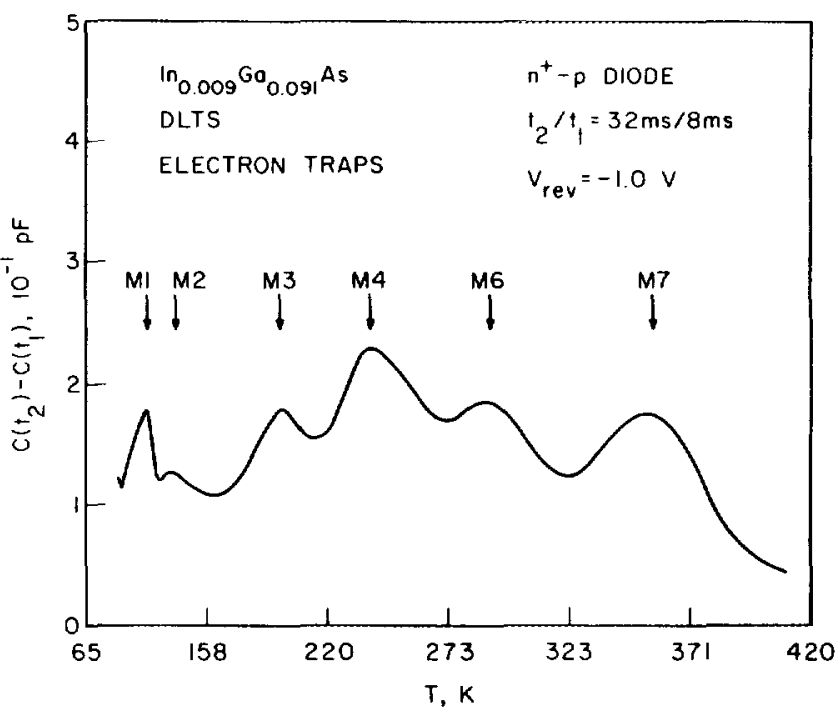

FIG. 1. Typical deep level transient spectroscopy data showing electron traps identified in molecular beam epitaxial (MBE) GaAs with $0.9 \%$ In doping. The data were recorded by applying a saturating injection pulse to a $n^{+}-p$ diode. 


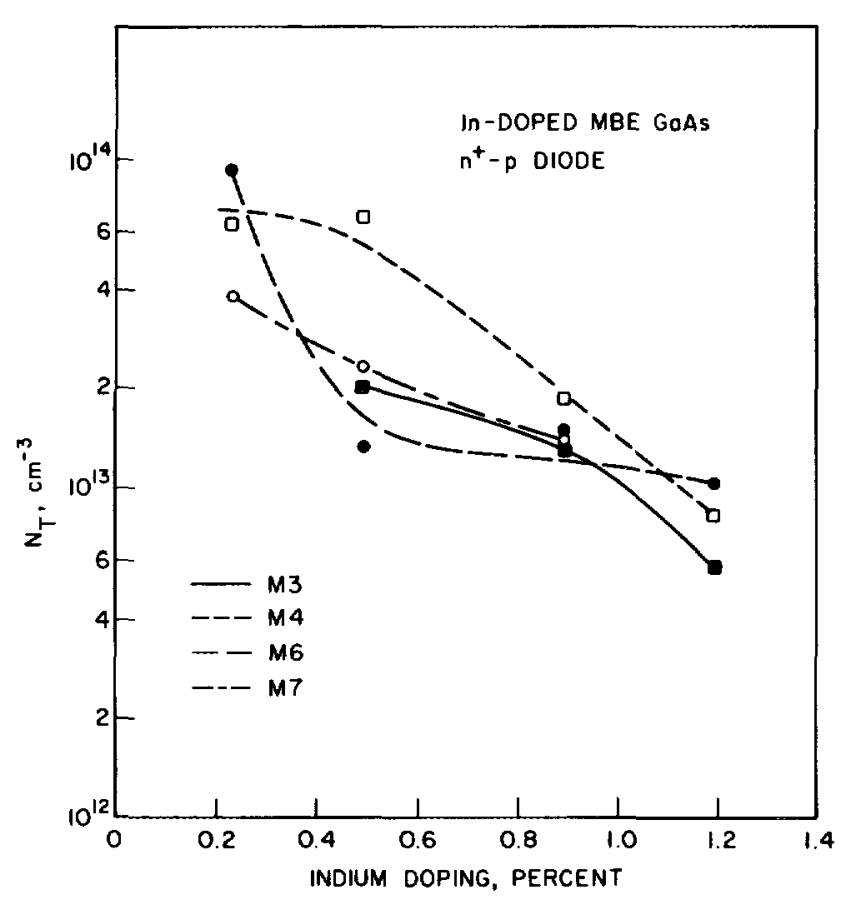

FIG. 2. Decrease in concentration of dominant electron traps with increase in In content of MBE GaAs.

surements showed almost negligible and nonreproducible peaks due to hole traps. We therefore chose to study the behavior of the dominant electron traps with addition of In. DLTS measurements were made on $n^{+}-p$ junction diodes using a 1-MHz capacitance meter. A saturation injection pulse of $\sim 20 \mathrm{~mA}$ was applied to the quiescent reverse biased diode $(4-10 \mathrm{~V})$ and its duration was typically $8-10 \mathrm{~ms}$. The temperature of the samples was varied in the range 77-420 K.

Typical DLTS data for a sample with $0.9 \%$ In in the solid solution are shown in Fig. 1. The absence of significant negative-going peaks confirms the absence of a large density of hole traps. Each positive-going peak corresponds to an electron trap level. The traps have been labeled in accordance with the original identification by Lang et al. ${ }^{12}$ All the traps have concentrations of the order of $1 \times 10^{13} \mathrm{~cm}^{-3}$, as found by Skromme et al. ${ }^{15}$ In addition to the traps $M 1, M 3$, and $M 4$, centers similar to those previously labeled $M 6$ and $M 7$ are also present in slightly lower concentrations. As pointed out by Lang et al. ${ }^{12}$ these may arise from impurities in the growth system or from the In itself. In this study we have chosen to investigate the behavior of the levels $M 3, M 4$, $M 6$, and $M 7$, since they are most prominent. The characterization of $M 1$ was limited by the low end temperature. At least two of these, $M 3$ and $M 4$, are known to be dominant in layers grown with $A s_{4}$ species and under As-stabilized conditions. The variation of the density of these four centers with increase in In content is shown in Fig. 2. We have confirmed that the trap densities in undoped GaAs grown under similar conditions are $(1-4) \times 10^{14} \mathrm{~cm}^{-3}$.

Low-temperature photoluminescence spectra of Indoped GaAs resemble those of undoped GaAs. In particular, we are interested in the additional defect-related sharpline emissions which occur at lower energies to the exciton peaks. ${ }^{14-19}$ In this study we report the behavior of the strongest emission peak, g. In our In-doped samples, this peak occurs at an energy of $1.509 \mathrm{eV}$ in spectra recorded at $11 \mathrm{~K}$. Figure 3 shows the initial sharp decrease and an eventual

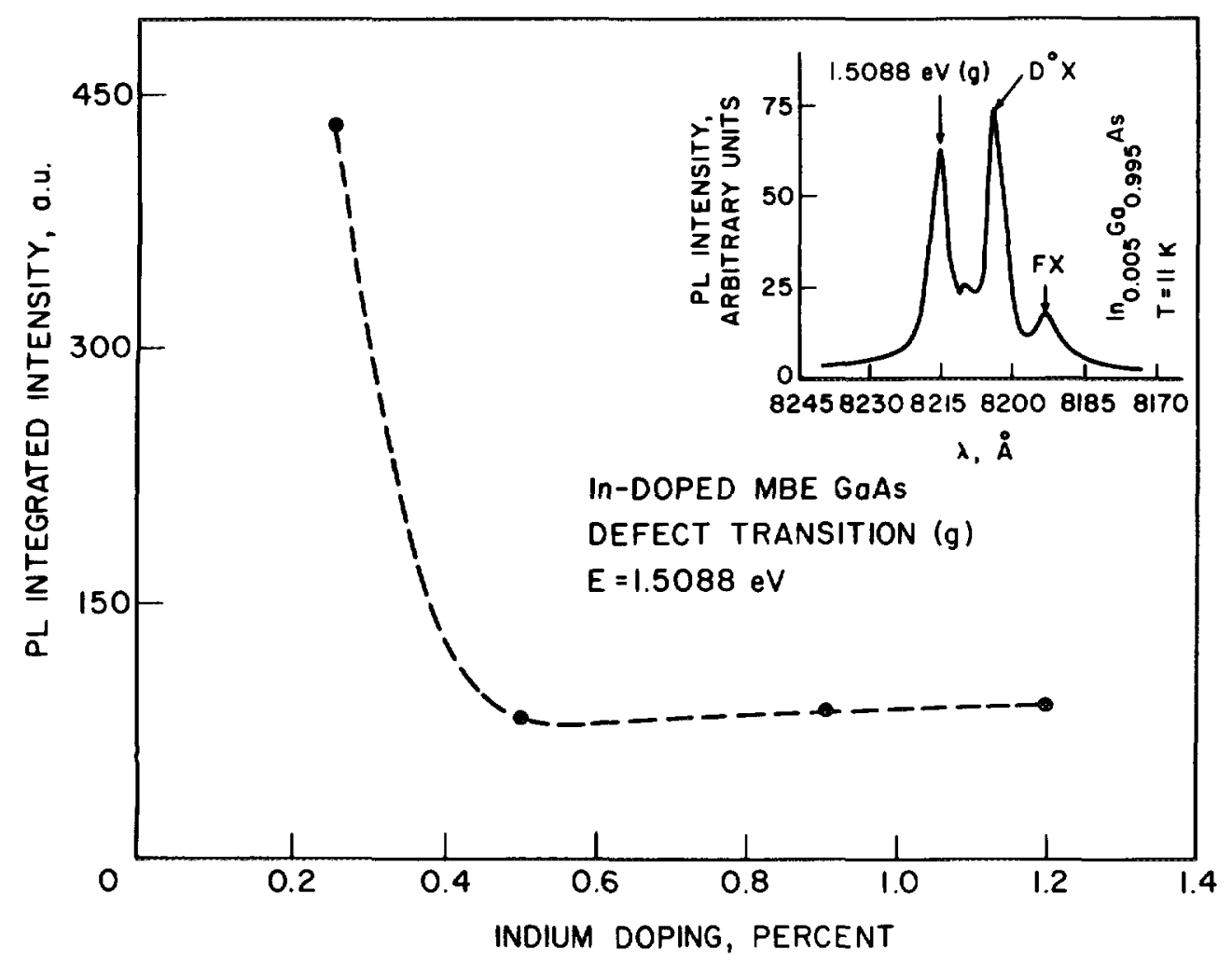

FIG. 3. Decrease in the integrated photoluminescence intensity of a prominent defect-related exciton peak $(g)$ with increasing In doping of undoped p-type GaAs single layers grown on undoped semi-insulating substrates. The inset shows a typical edge luminescence spectrum of an InGaAs sample grown on a semi-insulating substrate. 
leveling of the integrated intensity of this transition with increase of In content. In fact, the other transitions, which are weaker, could not be detected in the spectra for In content greater than $0.2 \%$ even with 20 times amplification in the recording system. It is important to note that the decrease is not caused by strain in the sample. This strain for In content $\sim 1.0 \%$ or less is expected to be extremely small and coherently distributed in the epitaxial film. Furthermore, Beneking $e t$ al. ${ }^{10}$ have recently shown an increase in the edge luminescence intensity by In doping of liquid phase epitaxial (LPE) GaAs.

Our present data seem to suggest that the deep level electron traps are probably native defects or complexes involving native defects. We believe these defects are Ga vacancies, since according to the expected growth kinetics such vacancies can be partially filled by In atoms, thereby lowering the trap densities. This conclusion agrees with that of Skromme et al. ${ }^{15}$ Our observations in the photoluminescence data also strongly support the fact that the observed exciton transition $g$ in our samples originates from native defects (possibly $V_{\mathrm{Ga}}$ ) or complexes involving native defects. This is in accordance with the hypothesis and observations of Briones and Collins ${ }^{17}$ and Skromme et al..$^{15}$ that these emissions originate from complexes of native defects and $\mathrm{C}$ acceptors. It should be pointed out that the peak intensities of the free- and donor- and acceptor-bound transitions also decrease slightly upon addition of In. This result indicates that a reduction of dislocation density, as observed by Beneking et $a l .{ }^{10}$ in the case of LPE GaAs, is not a plausible mechanism for In-doped MBE GaAs. In the latter case a large increase of edge luminescence intensity was observed. The decrease observed in our samples may arise from impurities in the In, in spite of using the purest material commercially available.
Useful discussions with Professor J. Singh and help in photoluminescence measurements provided by N. Debbar are gratefully acknowledged. The work was supported by NASA, Langley Research Center, under grant NAG-1-555.

'H. M. Hobgood, R. N. Thomas, D. L. Barrett, G. W. Elridge, M. M. Sopira, and M. C. Driver, in Proceedings of the International Conference on Semi-Insulating III-V Materials, Kah-nee-ta, 1984, edited by D. C. Look and J. S. Blakemore (Shiva, England, 1984), p. 149.

${ }^{2} \mathbf{M}$. Duseaux and S. Martin, in Proceedings of the International Conference on Semi-Insulating III-V Materials, Kah-nee-ta, 1984, edited by D. C. Look and J. S. Blakemore (Shiva, England, 1984), p. 111.

${ }^{3}$ W. C. Mitchel and P. W. Yu, J. Appl. Phys. 57, 623 (1985).

${ }^{4} H$. Ehrenreich and J. P. Hirth, Appl. Phys. Lett. 46, 668 (1985).

${ }^{5}$ H. V. Winston, A. T. Hunter, H. M. Olsen, R. P. Bryan, and R. E. Lee, Appl. Phys. Lett. 45, 447 (1984).

'H. Yamazaki, T. Honda, S. Ishida, and Y. Kawasaki, Appl. Phys. Lett. 45, 1109 (1984).

${ }^{7}$ P. Narozny and H. Beneking, Electron. Lett. 21, 1050 (1985).

${ }^{8} \mathrm{H}$. Beneking and N. Emeis, IEEE Electron Device Lett. EDL-7, 98 (1986).

${ }^{9} H$. Beneking, P. Narozny, P. Roentgen, and M. Yoshida, IEEE Electron Device Lett. EDL-7, 101 (1986).

${ }^{10} H$. Beneking, P. Narozny, and N. Emeis, Appl. Phys. Lett. 47, 828 (1985)

"J. Singh, Presented at the 13th Conference on the Physics and Chemistry of Semiconductor Interfaces, Pasadena, 1986.

${ }^{12}$ D. V. Lang, A. Y. Cho, A. C. Gossard, M. Ilegems, and W. Wiegmann, J. Appl. Phys. 47, 2558 (1976).

${ }^{13}$ P. Blood and J. J. Harris, J. Appl. Phys. 56, 993 (1984)

${ }^{14}$ P. K. Bhattacharya, H-J. Buhlmann, M. Ilegems, and J. L. Staehli, J. Appl. Phys. 53, 6391 (1982).

${ }^{15}$ B. J. Skromme, S. S. Bose, B. Lee, T. S. Low, T. R. Lepkowski, R. Y. DeJule, G. E. Stillman, and J. C. M. Hwang, J. Appl. Phys. 58, 4685 (1985).

${ }^{16}$ H. Kunzel and K. Ploog, Appl. Phys. Lett. 37, 416 (1980).

${ }^{17}$ F. Briones and D. M. Collins, J. Electron. Mater. 11, 847 (1982).

${ }^{18}$ P. J. Dobson, G. B. Scott, J. V. Neave, and B. A. Joyce, Solid State Commun. 43, 917 (1982).

${ }^{19}$ P. M. Petroff, C. Weisbuch, R. Dingle, A. C. Gossard, and W. Wiegmann, Appl. Phys. Lett. 38, 965 (1981). 